\title{
Cockroach sensitivity in allergic rhinitis patients; is it significant? To see prevalence of cockroach sensitivity in allergic rhinitis patients in Kingston area
}

\author{
Tahira Batool ${ }^{*}$, Rozita Borici-Mazi \\ From Canadian Society of Allergy and Clinical Immunology Annual Scientific Meeting 2010 \\ Victoria, Canada. 3-6 November 2010
}

\section{Background}

Role of cockroach allergy in asthma has been widely studied and the effect of environmental control on asthma symptoms has been established. However, the role of cockroach sensitivity remains unknown. We have designed this study to establish role of cockroach sensitization on allergic rhinitis.

\section{Hypothesis}

Cockroach allergy has significant role in allergic rhinitis.

\section{Population}

Allergic rhinitis patients attending allergy and clinical immunology clinic under Dr Rozita Borici-Mazi in Kingston General Hospital, Kingston ON.

\section{Method}

Retrospective chart review of patients evaluated for allergic rhinitis and underwent skin prick testing. A cohort of 250 patients was randomly selected with inclusion criteria being symptomatic allergic rhinitis and positive allergy skin prick testing to usual panel of allergens. Data collection included demographics, smoking exposure, symptom pattern, presence or absence of non-nasal symptoms, positive skin prick testing for cockroach and other environmental allergens such as dust mite, cat, dog, and seasonal pollens.

\footnotetext{
* Correspondence: 7TB11@queensu.ca Department of Internal Medicine, Queen's University, Kingston, Ontario, Canada
}

\section{Results}

Allergy to seasonal allergens was found to be the most common $(\mathrm{n}=191,76.4 \%)$ followed by house dust mite $(\mathrm{n}=149,59.6 \%)$ and cat allergen $(\mathrm{n}=118,47.2 \%)$. Cockroach sensitization was found in $62(25 \%)$. Among the cockroach sensitivity group, 8 patients had monosensitization to cockroach. All of them had perennial symptoms. $75 \%$ of these people were residents of urban areas. Two patients who had symptoms for more than 8 years had developed asthma.

\section{Conclusion}

Cockroach allergy is found to be one of the significant indoor allergens in allergic rhinitis in Kingston area. Given the relationship of Allergic Rhinitis and Asthma development, there is need to recognize this important allergen earlier and treat it through allergen avoidance and/or Immunotherapy, not only to treat allergic rhinitis symptoms but also to prevent development of allergic asthma. Further studies to establish the correlation between allergic rhinitis and cockroach sensitization are needed.

Published: 4 November 2010

doi:10.1186/1710-1492-6-S2-P11

Cite this article as: Batool and Borici-Mazi: Cockroach sensitivity in allergic rhinitis patients; is it significant? To see prevalence of cockroach sensitivity in allergic rhinitis patients in Kingston area. Allergy, Asthma \& Clinical Immunology 2010 6(Suppl 2):P11. 